\title{
Ensemble prediction and the role of higher order moments in atmospheric regime transitions
}

\author{
Terence J. O'Kane ${ }^{1} \quad$ Jorgen S. Frederiksen ${ }^{2}$
}

(Received 07 August 2006; revised 20 April 2007)

\begin{abstract}
We investigate the role of higher order moments in ensemble prediction studies using bred initial perturbations, ensemble averaged direct numerical simulations (DNS), and an inhomogeneous statistical closure model for 2D turbulence interacting with Rossby waves, mean fields and topography. Our closure methodology extends the previous work of Epstein [1969, Tellus 21] and Fleming [1971, Mon. Wea. Rev. 99], based on a cumulant discard hypothesis, to include all moments higher than second order. Unlike previous quasi-normal closures the quasi-diagonal direct interaction approximation (QDIA) model does not need to be Markovianized to guarantee positivity of energy and enstrophy and is valid for both strongly non-Gaussian and
\end{abstract}

See http://anziamj.austms.org.au/ojs/index.php/ANZIAMJ/article/view/74 for this article, (c) Austral. Mathematical Soc. 2007. Published April 30, 2007. ISSN $1446-8735$ 
strongly inhomogeneous flows. In addition the QDIA does not become unbounded for long integration periods as is the case for the eddy damped quasi-normal method [Fleming, 1971, Mon. Wea. Rev. 99]. We compare the QDIA to DNS for Northern Hemisphere flow during early November 1979 when a large and persistent high-low blocking dipole formed over the Gulf of Alaska. The formation and decay of atmospheric blocking events has typically been associated with a loss of predictability induced by large scale flow instabilities. Results from the QDIA statistical closure and DNS are shown to be in close agreement even throughout periods of block formation. We also examine distinct regimes of error growth arising from second and higher order effects.

\section{Contents}

\section{Introduction}

2 Barotropic flow on a $\beta$-plane

3 The closure equations

4 Diagnostics

5 Error growth during blocking

6 Ensemble prediction

7 Conclusions 


\section{Introduction}

The skill of numerical weather forecasts is determined by the instability properties of the atmospheric flow, by analysis errors and by model deficiencies. Early attempts at establishing the theoretical limits to atmospheric predictability focused on error growth in deterministic forecasts with the error determined from the divergence of pairs of initially close states [1, 14]. However, it was realised that weather forecasting should be regarded as a statistical problem of predicting the probability density function of atmospheric states or of calculating the moments of meteorological variables. Stochastic dynamic prediction methods [2] were developed in order to consider the effect of initial uncertainties on solving prognostic equations. This approach enables the gathering of information on the error or uncertainty in the forecast. Errors in the initial conditions arise through the impracticality of observing the atmosphere in sufficient detail with the result that deterministic forecasts fail over reasonable prediction periods. Stochastic methods [2] were applied in an effort to incorporate probabilistic information into a forecast through the direct forecast of mean and variance information obtained by solving inhomogeneous models of the statistical hydrodynamics. In addition to being impractical for large degrees of freedom these methods typically discard the third and higher order moments.

Subsequently closure schemes based on the quasi-normal [10] and the eddy-damped quasi-normal (EDQN) [13] hypotheses as well as Monte Carlo (MC) based ensemble prediction and deterministic methods were compared to the stochastic dynamic model in low resolution barotropic model studies [3]. It was shown that the stochastic dynamic model without moments higher than second order was only valid for very short range predictions of the order of a few days. In the EDQN model the three-point non-Gaussian correlations are empirically damped as an approximation to the natural damping effect that inclusion of all the higher order moments would introduce. At the time that these stochastic dynamic and EDQN prediction studies were performed Leith [8], Leith and Kraichnan [9], and Herring et al. [7] carried 
out analogous homogeneous predictability studies using more fundamental statistical closure methods.

This article demonstrates how the recent statistical closure models developed by Frederiksen [4], O'Kane and Frederiksen [11] (hereafter OF2004) and Frederiksen and O'Kane [6] (hereafter FO2005) offer a means by which to quantify the relative importance of flow inhomogeneities and non-Gaussian correlations. The quasi-diagonal direct interaction (QDIA) closure equations were derived by Frederiksen [4] for general barotropic mean flows interacting with inhomogeneous turbulence over topography on an $f$-plane. OF2004 tested the performance of the closure including cumulant update and regularised variants while the generalisation to Rossby wave turbulence on a $\beta$-plane was formulated and tested by FO2005. This inhomogeneous closure approach enables an improvement not just on the third moment discard hypothesis but is realisable and therefore does not become unstable for long run times unlike the EDQN closures [3].

In ensemble prediction studies, independently perturbed initial conditions are generated such that the fast growing errors in the analysis are approximately represented. Toth and Kalnay [15] developed a method for generating ensemble perturbations that lead to improved mean forecasts. They perturb the central forecast with initial random perturbations that they then integrate forward and rescale periodically to small amplitude. The perturbations thus effectively evolve according to an implicit tangent linear equation. This method of bred perturbations allows information about the fast growing errors to be incorporated into the initial conditions for the forecast. For particularly dynamic flows, such as when emergent coherent structures are developing, errors arise due to fast growing large scale instabilities. Bred vectors are stochastically and nonlinearly modified versions of the leading Lyapunov vectors (LLVs); thus, after an initial transient period $(\approx 7$ days for atmospheric flows) random perturbations tend to converge on the structure of the LLVs. As the perturbations are modified using a global (or regional) scaling factor the error growth associated with the evolving state of the model 
perturbation field develops within the breeding cycle and subsequently dominates forecast error growth.

In this article we examine perturbation or 'error' growth during a period in which a strong atmospheric high low blocking dipole formed over the Gulf of Alaska around the 5th November 1979. Our ensemble prediction study includes the regime transition from strongly zonal to blocked flow including the growing instabilities associated with block formation and decay. The inhomogeneous closure allows the quantification of error growth during transition between two different error growth regimes: the first corresponds to a regime where initial non-Gaussian effects are small in amplitude but cumulatively important, the second regime occurs as transient errors grow rapidly, eventually saturating with non-Gaussian effects becoming increasingly important. Throughout the closure calculations are regularised in the manner of of2004 in order that the effect of all higher order moments are correctly represented.

Section 2 briefly describes the spectral barotropic vorticity equation for turbulent flow over topography with Rossby waves on a generalised $\beta$-plane and in the presence of a large scale flow $U$. Section 3 states the functional form of the QDIA closure equations. The diagnostics are defined in Section 4. Section 5 outlines our approach to ensemble prediction using the method of bred perturbations. Section 6 compares closure and ensemble averaged direct numerical simulations (DNS) for an error growth study with a 5 day breeding cycle followed by a 5 day forecast period. Our conclusions are contained in Section 7.

\section{Barotropic flow on a $\beta$-plane}

We base our studies of both ensemble prediction and the formulation of statistical dynamical prediction on the generalised $\beta$-plane barotropic model for flow over topography. A more detailed explanation of the generalised $\beta$-plane, conserved quantities, the statistical mechanical equilibrium theory 
and nonlinear stability theory for flow on the generalised $\beta$-plane can be found in FO2005. As noted there, the full streamfunction is written in the form $\Psi=\psi-U y$, where $U$ is a large scale east-west flow and $\psi$ represents the 'small scales'. The evolution equation for the two-dimensional motion of the small scales over a mean topography is then described by the barotropic vorticity equation

$$
\frac{\partial \zeta}{\partial t}=-J\left(\psi-U y, \zeta+h+\beta y+k_{0}^{2} U y\right)+\hat{\nu} \nabla^{2} \zeta+f^{0} .
$$

Here $f^{0}$ is the forcing, $\hat{\nu}$ is the viscosity, and

$$
J(\psi, \zeta)=\frac{\partial \psi}{\partial x} \frac{\partial \zeta}{\partial y}-\frac{\partial \psi}{\partial y} \frac{\partial \zeta}{\partial x}
$$

is the Jacobian. The vorticity is the Laplacian of the streamfunction: $\zeta=$ $\nabla^{2} \psi$. The scale height for the topography $h=2 \mu g A H /\left(R T_{0}\right)$ where $H$ is the height of the topography, $R=287 \mathrm{~J} \mathrm{~kg}^{-1} \mathrm{~K}^{-1}$ is the gas constant for air, $T_{0}=273 \mathrm{~K}$ is the horizontally averaged global surface temp, $g$ is the acceleration due to gravity, $\mu=\sin \phi, \phi$ is the latitude and $A=0.8$ is the value of the vertical profile factor.

The term $k_{0}^{2} U y$ generalises the standard $\beta$-plane by the inclusion of an effect corresponding to the solid body rotation vorticity in spherical geometry where $k_{0}$ is a wave number that specifies the strength of this large scale vorticity. FO2005 noted that this additional small term results in a one-to-one correspondence between the dynamical equations, Rossby wave dispersion relations, nonlinear stability criteria and canonical equilibrium theory on the generalised $\beta$-plane and on the sphere.

The barotropic vorticity equation and the form-drag equation for $U$ are nondimensionalised by introducing the length and time scales $a / 2$, where $a$ is the earth's radius, and $\Omega^{-1}$, the inverse of the earth's angular velocity. With this scaling we consider flow on the domain $0 \leq x \leq 2 \pi, 0 \leq y \leq 2 \pi$. With the inclusion of relaxation towards the state $\bar{U}$ the form drag equation takes 
the form

$$
\frac{\partial U}{\partial t}=\frac{1}{S} \int_{S} h \frac{\partial \psi}{\partial x} d S+\alpha(\bar{U}-U) .
$$

Here, $\alpha$ is a drag coefficient and $S$ is the area of the surface $0 \leq x \leq 2 \pi$, $0 \leq y \leq 2 \pi$. In the absence of forcing and dissipation, equations (2) and (3) together conserve kinetic energy and potential enstrophy.

We derive the corresponding spectral space equations by representing each of the 'small scale' terms by a Fourier series; for example,

$$
\zeta(\mathbf{x}, t)=\sum_{\mathbf{k}} \zeta_{\mathbf{k}}(t) \exp (i \mathbf{k} \cdot \mathbf{x})
$$

where

$$
\zeta_{\mathbf{k}}(t)=\frac{1}{(2 \pi)^{2}} \int_{0}^{2 \pi} d^{2} \mathbf{x} \zeta(\mathbf{x}, t) \exp (-i \mathbf{k} \cdot \mathbf{x}),
$$

and $\mathbf{x}=(x, y), \mathbf{k}=\left(k_{x}, k_{y}\right), k=\left(k_{x}^{2}+k_{y}^{2}\right)^{1 / 2}$ and $\zeta_{-\mathbf{k}}$ is conjugate to $\zeta_{\mathbf{k}}$. As noted in equation (4.1) of FO2005, the sums in the consequent spectral equations run over the set $\mathbf{R}$ consisting of all points in discrete wavenumber space except the point $(0,0)$. The form-drag equation for $U$ are written in the same form as for the small scales by defining suitable interaction coefficients, representing the large scale flow as a component with zero wavenumber and extending the sums over wavenumbers. Thus the spectral form of the barotropic vorticity equation including large and small scales is compactly written as

$$
\begin{gathered}
\left(\frac{\partial}{\partial t}+\nu_{0}(\mathbf{k}) k^{2}\right) \zeta_{\mathbf{k}}(t)=\sum_{\mathbf{p} \in \mathbf{T}} \sum_{\mathbf{q} \in \mathbf{T}} \delta(\mathbf{k}+\mathbf{p}+\mathbf{q})\left[K(\mathbf{k}, \mathbf{p}, \mathbf{q}) \zeta_{-\mathbf{p}} \zeta_{-\mathbf{q}}\right. \\
\left.+A(\mathbf{k}, \mathbf{p}, \mathbf{q}) \zeta_{-\mathbf{p}} h_{-\mathbf{q}}\right]+f_{\mathbf{k}}^{0},
\end{gathered}
$$

where $\mathbf{T}=\mathbf{R} \cup \mathbf{0}$ and the interaction coefficients are defined in FO2005.

Here the complex $\nu_{0}(\mathbf{k})$ is related to the viscosity $\hat{\nu}$ and the intrinsic Rossby wave frequency $\omega_{\mathbf{k}}$ by

$$
\nu_{0}(\mathbf{k}) k^{2}=\hat{\nu} k^{2}+i \omega_{\mathbf{k}}
$$


where

$$
\omega_{\mathbf{k}}=-\frac{\beta k_{x}}{k^{2}} .
$$

We define $\zeta_{-\mathbf{0}}=i k_{0} U, \zeta_{\mathbf{0}}=\zeta_{-\mathbf{0}}^{*}$ and introduce a term $h_{-\mathbf{0}}$ that we take to be zero but which could more generally be related to a large scale topography. Note that $U$ is real and we define $\zeta_{0}$ to be imaginary. This is done to ensure that all the interaction coefficients that we use are defined to be purely real. Also with $\bar{\zeta}_{\mathbf{0}}=-i k_{0} \bar{U}, f_{\mathbf{0}}^{0}$ and $\nu_{0}\left(\mathbf{k}_{0}\right)$ are defined by $f_{\mathbf{0}}^{0}=\alpha \bar{\zeta}_{\mathbf{0}}$ with $\nu_{0}\left(\mathbf{k}_{0}\right) k_{0}^{2}=\alpha$. We also consider the case where the bare forcing is replaced by a relaxation term of the form $S_{\mathbf{k}}(t)=\kappa\left(\zeta_{\mathbf{k}}^{\text {obs }}-\zeta_{\mathbf{k}}\right)$ where $\kappa$ is the strength of the relaxation and $\zeta_{\mathrm{k}}^{\mathrm{obs}}$ are linearly interpolated daily observed fields.

\section{The closure equations}

Consider an ensemble of flows satisfying the generalised spectral barotropic vorticity equation (6). We express the vorticity $\zeta_{\mathbf{k}}$ and forcing $f_{\mathbf{k}}^{0}$ in terms of their ensemble average means, denoted by \langle\rangle , and the deviations from the means, denoted by ${ }^{\wedge}$ :

$$
\zeta_{\mathbf{k}}=\left\langle\zeta_{\mathbf{k}}\right\rangle+\hat{\zeta}_{\mathbf{k}}, \quad f_{\mathbf{k}}^{0}=\left\langle f_{\mathbf{k}}^{0}\right\rangle+\hat{f}_{\mathbf{k}}^{0} .
$$

The equation for the first moment or ensemble mean,

$$
\begin{aligned}
& \left(\frac{\partial}{\partial t}+\nu_{0}(\mathbf{k}) k^{2}\right)\left\langle\zeta_{\mathbf{k}}\right\rangle=\sum_{\mathbf{p}} \sum_{\mathbf{q}} \delta(\mathbf{k}+\mathbf{p}+\mathbf{q}) \\
& {\left[K(\mathbf{k}, \mathbf{p}, \mathbf{q})\left\{\left\langle\zeta_{-\mathbf{p}}\right\rangle\left\langle\zeta_{-\mathbf{q}}\right\rangle+C_{-\mathbf{p},-\mathbf{q}}(t, t)\right\}+A(\mathbf{k}, \mathbf{p}, \mathbf{q})\left\langle\zeta_{-\mathbf{p}}\right\rangle h_{-\mathbf{q}}\right]} \\
& +\left\langle f_{\mathbf{k}}^{0}\right\rangle,
\end{aligned}
$$

depends on the two-point cumulant

$$
C_{-\mathbf{p},-\mathbf{q}}(t, s)=\left\langle\hat{\zeta}_{-\mathbf{p}}(t) \hat{\zeta}_{-\mathbf{q}}(s)\right\rangle .
$$


Thus, from equation (10) see that to determine the mean field we need an equation for the two-point cumulant $C_{-\mathbf{p},-\mathbf{q}}(t, t)$. Similarly the second order cumulant requires knowledge of the third order cumulant which in turn depends on the fourth and so on. We are consequently faced with two problems, namely, the cost of computing the full covariance matrix, which is prohibitive at any reasonable resolution, and secondly the moment closure problem.

The second order expression for the diagonal two-time and single cumulants is expressed in terms of two- and three-point cumulants as

$$
\begin{aligned}
\left(\frac{\partial}{\partial t}+\nu_{0}(\mathbf{k}) k^{2}\right) C_{\mathbf{k}}\left(t, t^{\prime}\right) & =N_{\mathbf{k}}\left(t, t^{\prime}\right), \\
\left(\frac{\partial}{\partial t}+2 \Re \nu_{0}(\mathbf{k}) k^{2}\right) C_{\mathbf{k}}(t, t) & =2 \Re N_{\mathbf{k}}(t, t),
\end{aligned}
$$

where it is also convenient to separate the enstrophy production into the contributions from the two-point (inhomogeneous) and three-point (nonGaussian) terms:

$$
\begin{aligned}
N_{\mathbf{k}}\left(t, t^{\prime}\right)= & N_{\mathbf{k}}^{I}\left(t, t^{\prime}\right)+N_{\mathbf{k}}^{S}\left(t, t^{\prime}\right) \\
N_{\mathbf{k}}^{I}\left(t, t^{\prime}\right)= & \sum_{\mathbf{p}} \sum_{\mathbf{q}} \delta(\mathbf{k}+\mathbf{p}+\mathbf{q})\left\{A(\mathbf{k}, \mathbf{p}, \mathbf{q}) C_{-\mathbf{p},-\mathbf{k}}\left(t, t^{\prime}\right) h_{-\mathbf{q}}\right. \\
& \left.+K(\mathbf{k}, \mathbf{p}, \mathbf{q})\left[\left\langle\zeta_{-\mathbf{p}}(t)\right\rangle C_{-\mathbf{q},-\mathbf{k}}\left(t, t^{\prime}\right)+C_{-\mathbf{p},-\mathbf{k}}\left(t, t^{\prime}\right)\left\langle\zeta_{-\mathbf{q}}(t)\right\rangle\right]\right\} \\
N_{\mathbf{k}}^{S}\left(t, t^{\prime}\right)= & \sum_{\mathbf{p}} \sum_{\mathbf{q}} \delta(\mathbf{k}+\mathbf{p}+\mathbf{q}) K(\mathbf{k}, \mathbf{p}, \mathbf{q})\left\langle\hat{\zeta}_{-\mathbf{p}}(t) \hat{\zeta}_{-\mathbf{q}}(t) \hat{\zeta}_{-\mathbf{k}}\left(t^{\prime}\right)\right\rangle \\
& +\left\langle\hat{f}_{\mathbf{k}}^{0}(t) \hat{\zeta}_{\mathbf{k}}\left(t^{\prime}\right)\right\rangle
\end{aligned}
$$

Here we use the abbreviation $C_{\mathbf{k}}\left(t, t^{\prime}\right)=C_{\mathbf{k},-\mathbf{k}}\left(t, t^{\prime}\right)$ and $\left\langle\hat{f}_{\mathbf{k}}^{0}(t) \hat{\zeta}_{\mathbf{k}}\left(t^{\prime}\right)\right\rangle$ is a forcing term (see equation (19)). The off-diagonal elements of the response function, which measures the change in the vorticity perturbation due to an infinitesimal change in the forcing, is

$$
R_{\mathbf{k}, \mathbf{l}}\left(t, t^{\prime}\right)=\left\langle\frac{\delta \hat{\zeta}_{\mathbf{k}}(t)}{\delta \hat{f}_{\mathbf{l}}^{0}\left(t^{\prime}\right)}\right\rangle,
$$


so that the second order expression for the evolution of the diagonal two-time response function takes the form

$$
\begin{aligned}
& \left(\frac{\partial}{\partial t}+\nu_{0}(\mathbf{k}) k^{2}\right) R_{\mathbf{k}}\left(t, t^{\prime}\right) \\
= & \sum_{\mathbf{p}} \sum_{\mathbf{q}} \delta(\mathbf{k}+\mathbf{p}+\mathbf{q}) A(\mathbf{k}, \mathbf{p}, \mathbf{q}) R_{-\mathbf{p}, \mathbf{k}}\left(t, t^{\prime}\right) h_{-\mathbf{q}} \\
& +\sum_{\mathbf{p}} \sum_{\mathbf{q}} \delta(\mathbf{k}+\mathbf{p}+\mathbf{q}) K(\mathbf{k}, \mathbf{p}, \mathbf{q}) \\
& \times\left[\left\langle\zeta_{-\mathbf{p}}(t)\right\rangle R_{-\mathbf{q}, \mathbf{k}}\left(t, t^{\prime}\right)+R_{-\mathbf{p}, \mathbf{k}}\left(t, t^{\prime}\right)\left\langle\zeta_{-\mathbf{q}}(t)\right\rangle\right],
\end{aligned}
$$

where $R_{\mathbf{k}}\left(t, t^{\prime}\right)=R_{\mathbf{k}, \mathbf{k}}\left(t, t^{\prime}\right)$ with $R_{\mathbf{k}}(t, t)=1$ and $R_{\mathbf{k}}\left(t, t^{\prime}\right)=0$ for $t<t^{\prime}$. We may now express

$$
\left\langle\hat{f}_{\mathbf{k}}^{0}(t) \hat{\zeta}_{\mathbf{k}}\left(t^{\prime}\right)\right\rangle=\int_{t_{0}}^{t^{\prime}} d s F_{\mathbf{k}}^{0}(t, s) R_{-\mathbf{k}}\left(t^{\prime}, s\right)
$$

where $F_{\mathbf{k}}^{0}(t, s)=\left\langle\hat{f}_{\mathbf{k}}^{0}(t) \hat{f}_{\mathbf{k}}^{0 *}(s)\right\rangle$ is the variance of the random forcing.

The quasi-diagonal DIA closure equations overcome the problem of computational cost by expressing the off-diagonal two-point cumulant and response functions and higher order cumulants in terms of the diagonal elements. The resulting equations for the mean field, two-point cumulant and response functions are then expressed entirely as functionals of the diagonal elements of the two-point cumulant and response functions. These QDIA equations are computationally much more efficient than the general inhomogeneous closure equations which are intractable at any reasonable resolution.

Specifically, we have the following functional forms:

$$
\begin{aligned}
C_{\mathbf{k},-1}\left(t, t^{\prime}\right) & \approx C_{\mathbf{k},-1}^{\mathrm{QDIA}}\left(t, t^{\prime}\right)\left[C_{\mathbf{k}}, R_{\mathbf{k}},\left\langle\zeta_{\mathbf{k}}\right\rangle, h_{\mathbf{k}}\right] \\
R_{\mathbf{k},-\mathbf{l}}\left(t, t^{\prime}\right) & \approx R_{\mathbf{k},-\mathbf{l}}^{\mathrm{QDIA}}\left(t, t^{\prime}\right)\left[C_{\mathbf{k}}, R_{\mathbf{k}},\left\langle\zeta_{\mathbf{k}}\right\rangle, h_{\mathbf{k}}\right] \\
\left\langle\hat{\zeta}_{\mathbf{k}}(t) \hat{\zeta}_{-\mathbf{l}}(t) \hat{\zeta}_{\mathbf{l}-\mathbf{k}}\left(t^{\prime}\right)\right\rangle & \approx\left\langle\hat{\zeta}_{\mathbf{k}}(t) \hat{\zeta}_{-\mathbf{l}}(t) \hat{\zeta}_{\mathbf{l}-\mathbf{k}}\left(t^{\prime}\right)\right\rangle^{\mathrm{QDIA}}\left[C_{\mathbf{k}}, R_{\mathbf{k}},\left\langle\zeta_{\mathbf{k}}\right\rangle, h_{\mathbf{k}}\right]
\end{aligned}
$$


The explicit expressions for $C_{\mathbf{k},-1}^{\mathrm{QDIA}}\left(t, t^{\prime}\right), R_{\mathbf{k},-\mathbf{l}}^{\mathrm{QDIA}}\left(t, t^{\prime}\right)$ and $\left\langle\hat{\zeta}_{\mathbf{k}}(t) \hat{\zeta}_{-\mathbf{l}}(t) \hat{\zeta}_{\mathbf{l}-\mathbf{k}}\left(t^{\prime}\right)\right\rangle^{\mathrm{QDIA}}$ and including an even more computationally efficient restart procedure can be found in OF2004 and FO2005.

\section{Diagnostics}

Next, we define a number of diagnostics that we employ in the following section for analysing the predictability studies. We define the zonally averaged transient (error) $e^{T}\left(k_{x}, t\right)$ and mean $e^{M}\left(k_{x}, t\right)$ kinetic energy spectra by

$$
\begin{aligned}
e^{T}\left(k_{x}, t\right) & =\frac{1}{2} \sum_{\mathbf{k}_{y}}\left[C_{\mathbf{k}}(t, t)\right] / k^{2}, \\
e^{M}\left(k_{x}, t\right) & =\frac{1}{2} \sum_{\mathbf{k}_{y}}\left[\left\langle\zeta_{\mathbf{k}}(t)\right\rangle\left\langle\zeta_{-\mathbf{k}}(t)\right\rangle\right] / k^{2} .
\end{aligned}
$$

The kinetic energy of the large scale flow is plotted at $k_{x}=0$.

We also use a measure based on palinstrophy production to characterise the error growth during forecasts. For this it is useful to separate the enstrophy production terms as in equation (14) into the contribution from the two-point inhomogeneous $N^{I}(t, t)$, and three-point non-Gaussian $N^{S}(t, t)$, terms. The palinstrophy production measure based on the three-point nonGaussian terms is just the skewness $S^{K}$ that has been commonly used to examine the small scale behaviour of homogeneous [5] and inhomogeneous turbulence [11]. However, the current regime of small amplitude transient errors growing on larger amplitude mean flows differs from these previous studies in that most of the transfer results from the two-point inhomogeneous production. One of our aims is to quantify the relative contribution of the two-point inhomogeneous and three-point and higher order non-Gaussian terms to both the instantaneous and cumulative error growth. Examination of both the palinstrophy production and kinetic energy spectra allows this quantification. 
The palinstrophy production measure $P^{M}$ is defined in terms of the transient enstrophy $\hat{\mathcal{F}}$, transient palinstrophy $\hat{\mathcal{P}}$ and palinstrophy production $\mathcal{K}$ :

$$
\begin{aligned}
P^{M}(t) & =2 \mathcal{K} /\left(\hat{\mathcal{P}} \hat{\mathcal{F}}^{1 / 2}\right), \\
\hat{\mathcal{F}}(t) & =\frac{1}{2} \sum_{\mathbf{k}} C_{\mathbf{k}}(t, t), \\
\hat{\mathcal{P}}(t) & =\frac{1}{2} \sum_{\mathbf{k}} C_{\mathbf{k}}(t, t) k^{2}, \\
\mathcal{K}(t) & =\mathcal{K}^{I}(t)+\mathcal{K}^{S}(t)=\sum_{\mathbf{k}} k^{2} N_{k}^{I}(t, t)+\sum_{\mathbf{k}} k^{2} N_{k}^{S}(t, t) .
\end{aligned}
$$

\section{$5 \quad$ Error growth during blocking}

Our aim is to compare closure and ensemble averaged DNS results for a 5 day forecast starting after an initial 5 day breeding period using the closure to separate and quantify the various contributions to error growth with the DNS used as a measure of the accuracy of the closure. We focus on the ensemble predictability of $500 \mathrm{hPa}$ Northern Hemisphere atmospheric flows during a period in October and November 1979 in which a large scale blocking highlow dipole formed over the Gulf of Alaska on 5th November, amplified and persisted until 12th November and then weakened and moved downstream.

We examine the evolution of transient error fields in closure and DNS calculations on trajectories similar to that taken by the atmospheric $500 \mathrm{hPa}$ field between the 26th October and the 8th November 1979. For the mean fields within a barotropic model to closely follow an observed trajectory it is necessary to specify suitable time-evolving source terms. A relaxation term of the form $S_{\mathbf{k}}(t)=\kappa\left(\zeta_{\mathbf{k}}^{\text {obs }}-\zeta_{\mathbf{k}}\right)$ is added to the right-hand side of the spectral barotropic vorticity equation where $\zeta_{\mathbf{k}}^{\mathrm{obs}}$ are the linearly interpolated daily observed fields. We start the simulation with the observed $500 \mathrm{hPa}$ streamfunction for 1200 UTC on the 26th of October and use an $e$-folding 
relaxation time $\left(\kappa^{-1}\right)$ of 2 days over the 10 day period ( 5 day breeding, 5 day forecast). The source term is calculated at each time step of the unperturbed simulation, stored and then applied to both perturbed ensemble DNS runs and to the mean field equation of the closure. The Doppler frequency was treated perturbatively and we present results for $k_{0}^{2}=1 / 2$ and $\beta=1 / 2$ which has a dimensional value of $1.15 \times 10^{-11} \mathrm{~m}^{-1} \mathrm{~s}^{-1}$ and is typical of the $\beta$-effect at $60^{\circ}$ latitude. Throughout a viscosity of $2.5 \times 10^{5} \mathrm{~m}^{2} \mathrm{~s}^{-1}$ corresponding to a non-dimensional value of $\hat{\nu}=3.378 \times 10^{-4}$ is used. Both closure and DNS are run with a 1 hour time step. The initial errors fields, prior to breeding, have Gaussianly distributed isotropic spectra that are taken to be approximately constant with wave number. These initial perturbations are several orders of magnitude less than the mean field at all wave numbers. The DNS shows ensemble averaged results from 1800 simulations. All calculations are for circularly truncated $\mathbf{k}=16$ (C16) wavenumber space.

We take a continuous approach to breeding, that is, re-scaling after every evolved time-step. For atmospheric flows it is the large scales that are of primary importance in determining the dynamics and hence error growth; thus the global breeding scaling parameter is implemented in terms of streamfunction, that is,

$$
\begin{aligned}
g(t) & =\left[\frac{\sum_{\mathbf{k}} C_{\mathbf{k}}\left(t_{0}, t_{0}\right) / k^{4}}{\sum_{\mathbf{k}} C_{\mathbf{k}}(t, t) / k^{4}}\right]^{1 / 2}, \\
\hat{\zeta}_{\mathbf{k}}\left(t_{+}\right) & =g(t) \hat{\zeta}_{\mathbf{k}}\left(t_{-}\right), \\
C_{\mathbf{k}}\left(t_{+}, t_{+}\right) & =g(t) g(t) C_{\mathbf{k}}\left(t_{-}, t_{-}\right), \\
C_{\mathbf{k}}\left(t_{+}, t_{+}^{\prime}\right) & =g(t) g\left(t^{\prime}\right) C_{\mathbf{k}}\left(t_{-}, t_{-}^{\prime}\right),
\end{aligned}
$$

where - and + indicate prior and posterior fields. In both the DNs and closure the off-diagonal elements of the covariances are adjusted. In the DNS this occurs through direct application of the global scaling factor to the vorticity field, equation (29), whereas in the closure it occurs through the diagonal cumulant and is thus consistent with the assumptions of the QDIA.

After the breeding period the evolved error fields are prepared so that 
they contain information about the fastest growing errors (incorporated during the breeding cycle) arising due to instabilities present in the underlying dynamics of the flow. Thus the forecast errors contain, by construction, information about the underlying dynamics and are thus prepared to capture the behaviour of the fast growing error modes of the flow.

\section{Ensemble prediction}

Figure 1 compares the DNS and regularised QDIA calculations beginning on the 26th October 1979. Over both the initial 5 day breeding period, starting from isotropic initial perturbations (Figure 1a), and 5 day forecast we observe that at all times the mean kinetic energy spectra are in good agreement (Figure $1 \mathrm{~b}$ ) and that the bred perturbations are clearly "peaked" at $k=3$ which is the fastest growing scale. On day 10 it is evident that the error field has saturated at the small scales and hence small scale predictability is being lost. O'Kane and Frederiksen [12] compared similar calculations using cumulant discard and unregularised variants of the QDIA finding that omission of higher order non-Gaussian correlations leads to under-prediction of the evolved small scale error kinetic energy.

From the palinstrophy production measure, Figure 1d), observe that there broadly exists four distinct regimes in the error growth over the depicted 10 day experiment. The first two regimes, corresponding to the organisation and formation of coherent error structures, are contained within the 5 day breeding period starting on the 26th October 1979. During the first day of the breeding period there is a dramatic and rapid growth in the palinstrophy production measure $P^{M}$ as the initial error field evolves from Gaussian isotropic initial conditions towards the leading instability vectors associated with growth of the off-diagonal covariances. From approximately day 2 through to day 5 the palinstrophy production measure grows slows and its evolution is now largely determined by flow inhomogeneities. At day 5 the 

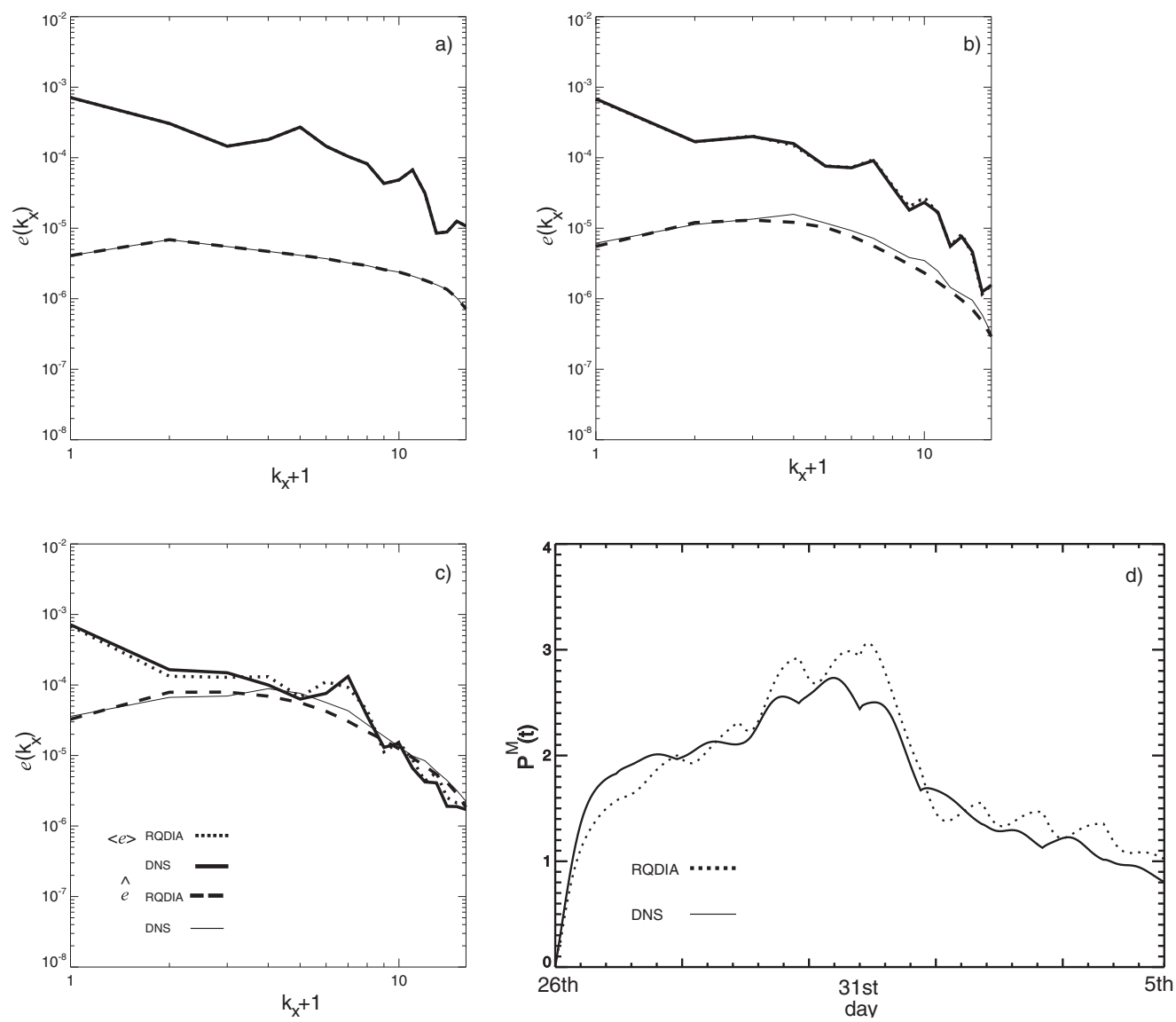

FiguRE 1: Bred perturbations are generated over a 5 day period starting on the 26 th October 1979 , followed by a 5 day forecast period. The initial, day 5 and day 10 mean and perturbation kinetic energy fields (non-dimensional) as a function of zonal wavenumber are shown for DNS and regularised QDIA calculations in figures a), b) and c) respectively. Figure d) shows the evolving palinstrophy production measure over the entire 10 day period. 
breeding ends and the forecast begins. The final two regimes occur between days 6 and 7 and from day 7 onwards. Bred initial forecast perturbations result in fast growing instability vectors at the large scales around day 6 where the error field amplitude is observed to undergo rapid increases, with a corresponding decrease in $P^{M}$. From day 7 onwards the growth of the error amplitude is much reduced as the errors start to saturate. Error saturation at the small scales occurs at about day 10 .

\section{Conclusions}

We have examined the role of higher order moments in ensemble prediction studies using the method of bred perturbations and compare a new statistical closure with ensemble averaged direct numerical simulations. The regularised QDIA closure extends previous statistical closures and stochastic dynamic methods by including all higher order moments. It thereby avoids many of the problems, such as under estimated forecast error variances, associated with methods based on a cumulant discard or quasi-normal hypothesis. Kinetic energy spectra have been used to show the close comparison between closure and DNS at the large scales. We also defined a palinstrophy production measure and employed it to show the close agreement between closure and DNS at the small scales. We identified four distinct regimes associated with error organisation and growth during a 10 day experiment consisting of 5 days of continuous breeding and 5 days of forecast. We consider a period during which a high-low blocking dipole was forming over the Gulf of Alaska in early November 1979 generating fast growing large scale flow instabilities. Additional studies [12], where non-Gaussian correlations and the two-point off-diagonal variances are removed, such as when a quasi-normal assumption is made, showed that error growth is severely underestimated resulting in poor predictability estimates. The QDIA closure allows the quantification of the relative contributions from inhomogeneous and non-Gaussian terms to the palinstrophy production. O'Kane and Frederiksen [12] showed that it is 
not until the error field saturates that instantaneous non-Gaussian correlations become significant; however, they have important cumulative effects on the small scale energy spectra even over short evolution periods.

\section{References}

[1] J. G. Charney 1966 The feasibility of a global observation and analysis experiment. Bull. Amer. Meteor. Soc. 47, 200-220. C52

[2] E. S. Epstein, Stochastic dynamic prediction. Tellus 21, 1969, 739-759. C52

[3] R. J. Fleming 1971 On stochastic dynamic prediction: I. The energetics of uncertainty and the question of closure. Mon. Wea. Rev. 99, 851-872, doi:10.1175/1520-0493(1971)099;0851:OSDP ¿2.3.CO;2 C52, C53

[4] J. S. Frederiksen 1999 Subgrid-scale parameterizations of eddy-topographic force, eddy viscosity, and stochastic backscatter for flow over topography. J. Atmos. Sci. 56, 1481-1494, doi:10.1175/1520-0469(1999)056;1481:SSPOET¿2.0.CO;2 C53

[5] J. S. Frederiksen and A. G. Davies 2000 Dynamics and spectra of cumulant update closures for two-dimensional turbulence. Geophys. Astrophys. Fluid Dyn. 92, 197-231. C60

[6] J. S. Frederiksen and T. J. O'Kane 2005 Inhomogeneous closure and statistical mechanics for Rossby wave turbulence over topography. J. Fluid Mech. 539, 137-165, doi:10.1017/S0022112005005562 C53

[7] J. R. Herring, J. J. Riley, G. S. Patterson Jr. and R. H. Kraichnan 1973 Growth of uncertainty in decaying isotropic turbulence. J. Atmos. Sci. 30, 997-1006, doi:10.1175/1520-0469(1973)030ز0997:GOUIDI ¿2.0.CO;2 C52 
[8] C. E. Leith 1971 Atmospheric predictability and two-dimensional turbulence. J. Atmos. Sci. 28, 145-161, doi:10.1175/1520-0469(1971)028;0145:APATDT¿2.0.CO;2 C52

[9] C. E. Leith and R. H. Kraichnan 1972 Predictability of turbulent flows. J. Atmos. Sci. 29, 1041-1058, doi:10.1175/1520-0469(1972)029¡1041:POTF ¿2.0.CO;2 C52

[10] M. Millionshtchikov 1941 On the theory of homogeneous isotropic turbulence. Compte Rendus (Doklady) de l'Academie Des Sciences de l'U.S.S.R. 32, 615-618. C52

[11] T. J. O'Kane and J. S. Frederiksen 2004 The QDIA and regularized QDIA closures for inhomogeneous turbulence over topography. J. Fluid Mech. 504, 133-165, doi:10.1017/S0022112004007980 C53, C60

[12] T. J. O'Kane and J. S. Frederiksen 2007 A comparison of statistical dynamical and ensemble prediction during blocking. J. Atmos. Sci. to appear. C63, C65

[13] S. A. Orszag 1970 Analytical theories of turbulence. J. Fluid Mech. 41, 363-386. C52

[14] J. Smagorinsky 1969 Problems and promises of deterministic extended range forecasting. Bull. Amer. Meteor. Soc. 50, 286-311. C52

[15] Z. Toth and E. Kalnay 1997 Ensemble forecasting at NCEP and the breeding method. Mon. Wea. Rev. 125, 3297-3319, doi:10.1175/1520-0493(1997)125;3297:EFANAT¿2.0.CO;2 C53 


\section{Author addresses}

1. Terence J. O'Kane, Antarctic Climate and Ecosystems Co-operative Research Centre, University of Tasmania, Australia. mailto:Terence.OKane@utas.edu.au

2. Jorgen S. Frederiksen, CSIRO Marine and Atmospheric Research, Aspendale, Australia. 